\section{PWE-042 PROFILE BIOMARKER: EFFECT OF STEROID TREATMENT ON A PROGNOSTIC GENE EXPRESSION SIGNATURE}

1,2Nurulamin Noor* 1,2James Lee, ${ }^{1}$ Daniele Biasci, ${ }^{1,2}$ Eoin McKinney, ${ }^{2}$ Konstantina Strongili,

1,2 Paul Lyons, ${ }^{1,2}$ Miles Parkes, ${ }^{1,2}$ Kenneth Smith. ${ }^{1}$ University of Cambridge School of Clinical Medicine, Cambridge, UK; ${ }^{2}$ Addenbrooke's Hospital, Cambridge University Hospitals NHS Trust, Cambridge, UK

\subsection{6/gutjpl-2018-BSGAbstracts. 174}

Background The course of Crohn's disease varies substantially between patients, but reliable prognostic markers are not available in clinical practice. This hinders disease management because patients with aggressive disease are undertreated by 'step-up' therapy, while those with indolent disease are exposed to risks of unnecessary immunosuppression if a 'top-down' approach is used. Previously, we have described a transcriptional signature that is detectable in whole blood at diagnosis, correlating with subsequent disease course. A biomarker-stratified trial in Crohn's disease (PROFILE trial) is currently underway, recruiting 400 newly diagnosed patients from approximately 50 centres in the UK, and stratifying their therapy using this biomarker. Currently, patients need to be off systemic corticosteroids at recruitment, as it is not known whether the results of the test remain reliable while on steroid treatment, which can affect gene expression. If the biomarker results were to be reliable despite systemic corticosteroids, this would enable the test to be used on patients who had already begun steroid therapy and would broaden the clinical utility.

Methods Four separate cohorts of IBD patients were recruited $(\mathrm{n}=10$ per cohort). From each patient a PAXgene RNA tube was collected, along with routine haematology and biochemistry tests, and a stool sample for faecal calprotectin. Detailed clinical phenotyping information was prospectively collected for at least 6 months. The four cohorts comprised: 1) patients admitted with acute severe flares of IBD with sample collection pre-intravenous steroid and at 3 further timepoints on IV steroid medication (day 3, day 5, week 6), 2) patients seen in outpatient clinic with flares of IBD - sample collection preoral steroid treatment and at 2 further timepoints on steroid medication (week 1, week 6), 3) recently diagnosed IBD patients ( $<3$ months since diagnosis) who had active inflammation despite ongoing steroid treatment, and 4) patients with established IBD with active inflammation on steroid treatment. RNA was extracted from PAXgene tubes, reverse-transcribed and RT-qPCR performed to detect the prognostic transcriptional signature. If detectable, the subsequent course of disease was assessed and compared between $\mathrm{IBD}^{\text {hi }}$ and $\mathrm{IBD}^{\text {lo }}$ groups. This data is currently being finalised and the results will be available at the BSG annual conference.

Conclusions We have developed, optimised and validated a whole blood qPCR classifier that is able to predict disease course from diagnosis in patients with Crohn's disease. This represents a major step towards personalised therapy and is currently being tested in the PROFILE trial, the first biomarker-stratified trial in inflammatory disease. The effect of steroid treatment on this biomarker signature has yet to be determined, but is an important step in order to maximise the clinical utility of this test and possibly widen inclusion of patients into the PROFILE trial.

\section{PWE-043 MANAGING ACUTE SEVERE COLITIS IN A DISTRICT} GENERAL HOSPITAL

Sophia Oxenburgh*, Christopher Wells. University Hospital Of North Tees, Gateshead, UK

\subsection{6/gutjnl-2018-BSGAbstracts. 175}

Introduction Following a critical incident in the care of a patient with acute severe colitis (ASC) we audited practice against the European Crohn's and Colitis Organisation (ECCO) standards for ASC. Performance was compared to the 2014 Inflammatory Bowel Disease (IBD) Audit. Since 2014 gastroenterology inpatient care changed to consultant of week instead of a single consultant. This improved discharge rates (top 4 in England for acute admission targets) but may have disrupted quality of care for more complex conditions like IBD.

Methods Adult coding database searched for 'colitis' from 01/ 01/2016-31/10/2017. Admissions $<48$ hours excluded. Discharge letters were searched for ASC cases and notes then reviewed. Admissions with ASC were audited against the ECCO standards.

Results

- 40 admissions with ASC (30 patients -2 had 3 and 8 had 2 admissions)

- 17 saw gastroenterologist day 1 (median day 2, range $1-4$ )

- 39 went to gastro ward

- 32 had abdominal xray on day 1

- 1 had flexi sig day 1, 12 had lower GI scope pre-admission, 18 during admission (median day 3, range 1-11) and 9 had none

- 34 had IV hydrocortisone on day 1 (median day 3, range 16)

- 26 had $\mathrm{Ca} /$ vit D

- All had low molecular weight heparin (LMWH)

- 14 saw dietician, 33 had MUST scored

- 24 saw IBD nurse

- 13 saw stoma nurse

- 6 saw surgeon on day 1 (median day 2, range 1-14) and 15 did not get referred

- 10 required surgery -7 done by a colorectal surgeon (6 laparoscopically, 4 open)

- Median surgery day 9 (range 2 - 23 from admission)

- 1 on biologic pre-admission

- 2 had surgery on readmission

- 3 based on clinical features

- 3 not responded to biologic

- 1 not clear

- Biologics given to 10 patients - 2 day 3, 1 day 4, 6 day 5 and 1 day 6 (2:8 between adalimumab:infliximab). No ciclosporin. $90 \%$ did not need surgery.

Conclusion In 2014 our trust data showed we performed on par with National audit.

In 2017 we were equivalent to or outperformed the National figures for:

- Care on a specialist ward (98\% vs 69\%)

- Nutritional assessment (80\% vs $82 \%)$

- Dietician review (45\% v 40\%)

- Prescribing LMWH (100\%)

- IBD nurse review (60\% vs 66\%) 
We performed below the IBD audit for:

- Sigmoidoscopy in 72 hours (28\% vs 99\%)

- Prescribing $\mathrm{Ca} /$ vit D (65\% vs $74 \%)$

- Median time to surgery (9 vs 7.5 days)

Important standards of IBD nurse and dietician review maintained. Delay in endoscopic evaluation and therefore time to surgery indicate there has been a slipping of standards in ASC care. This may be related to less direct ward continuity.

Our data show a drop in performance (access to endoscopy and time to surgery). They have allowed us to critically appraise our acute IBD service thus leading to care delivery change and an education package for medical and surgical directorates. A repeat audit is planned in 24 months to demonstrate quality improvement as a result of this.

\section{PWE-044 THE IBD BIORESOURCE: PROGRESSING FROM GENETICS TO FUNCTION AND CLINICAL TRANSLATION IN CD \& UC}

\footnotetext{
1,2 Laetitia Pele* ${ }^{*}$ 1,2Rachel Simpkins, ${ }^{1,2}$ Catherine Thorbinson, 1,2Deepthy Francis, 1,2Sophie Lewis, ${ }^{2}$ Rasha Shawky, ${ }^{1,2}$ Miles Parkes, ${ }^{1,2}$ UK IBD Genetic Consortium. ${ }^{1}$ Department of Medicine, University of Cambridge, UK, Cambridge, UK; ${ }^{2}$ IBD Bioresource, NIHR BioResource, Cambridge, UK, Cambridge, UK
}

\subsection{6/gutjnl-2018-BSGAbstracts. 176}

Introduction The Inflammatory Bowel Disease (IBD) BioResource was established by the UK IBD Genetics Consortium and the NIHR BioResource in 2016 to expedite the clinical translation of recent genetics advances and support important IBD research. It recruits patients from hospitals UK-wide, the aim being to enlist 25000 participants who can be further invited for future research studies based on genotype and/or phenotype. DNA samples will also be used in on-going IBD genetics analyses by the UK IBD Genetics Consortium.

Methods The Main cohort comprises individuals with established Crohn's Disease (CD) and Ulcerative Colitis (UC). Both clinical and self-reported phenotype data are collected, alongside plasma, serum and DNA samples for whole Genome Sequencing. The Inception cohort aims to recruit a sub-set of 1000 individuals newly diagnosed with IBD that will provide more detailed sampling, unconfounded by drug treatment or effects of surgery and includes stool, biopsy tissue and whole blood for RNA. This cohort offers a unique resource to undertake transcriptomic, meta-genomic, metabolomic and proteomic studies and facilitate research into determinants, predictors and biomarkers of IBD disease course and treatment response. In addition to Stage 1 recruitment, the IBD BioResource panel can be accessed by any researchers (both academic and commercial) with ethically approved proposals and may involve a range of possible options, such as access to data or samples or recall of genotype-selected participants to donate further samples or trial novel therapies.

Results 24 months in, we have 59 hospitals active and $\sim 30$ in set-up.

Main Cohort: over 8000 patients have been recruited - CD/ UC 4,390/3,832.

CD phenotypes: extent - 31\% ileal, 32\% ileo-colonic, 31\% colonic, $28 \%$ with peri-anal involvement; behaviour - $68 \%$ inflammatory, $21 \%$ stenosing and $11 \%$ penetrating.

$5048(61 \%)$ have been prescribed thiopurines and 2324 $(28 \%)$ required treatment cessation. $3842(47 \%)$ have received anti-TNF $\alpha$ therapy.
45\% CD and 5\% UC have required surgery. Most subjects are recruited in medical gastroenterology clinics so there are biases in this dataset. We will provide updates and breakdown of numbers at the meeting and discuss downstream stage 2 studies.

Inception Cohort: has been launched and is being rolled out nationally.

Conclusion The IBD BioResource and its network are on course to generate an accessible platform of patients and their data that will facilitate high quality translational IBD research.

\section{PWE-045 VEDOLIZUMAB RESULTS IN REDUCED HOSPITALISATION AND STEROID USE OVER 1-YEAR: RESULTS FROM THE SCOTTISH VEDOLIZUMAB COHORT}

${ }^{1}$ Nikolas Plevris*, ${ }^{1}$ Cher Chuah, ${ }^{1}$ Phil Jenkinson, ${ }^{2} \mathrm{R}$ Allen, ${ }^{2} \mathrm{P}$ Baker, ${ }^{3}$ Paul Brennan, ${ }^{1}$ Antonia Churchhouse, ${ }^{1} S$ Din, ${ }^{4}$ Emma Donoghue, ${ }^{2}$ Daniel Gaya, ${ }^{3}$ Max Groome, ${ }^{5} \mathrm{H}$ Jafferbhoy, ${ }^{1} \mathrm{G}$ Jones, ${ }^{2}$ Jonathan Macdonald, ${ }^{2}$ Morag MacMaster, ${ }^{3} \mathrm{Craig}$ Mowat, ${ }^{2} \mathrm{G}$ Naismith, ${ }^{1} \mathrm{C}$ Noble, ${ }^{6} \mathrm{~L}$ Potts, ${ }^{2} \mathrm{E}$ Saffouri, ${ }^{2} \mathrm{JP}$ Seenan, ${ }^{5} \mathrm{~A}$ Sengupta, ${ }^{1} \mathrm{Alan}$ Shand, ${ }^{3} \mathrm{P}$ Shasi, ${ }^{3} \mathrm{~J}$ Todd, ${ }^{2} \mathrm{~J}$ Veryan, ${ }^{6} \mathrm{~A}$ Watson, ${ }^{1} \mathrm{E}$ Watson, ${ }^{4} \mathrm{D}$ Watts, ${ }^{1} \mathrm{I}$ Arnott, ${ }^{1} \mathrm{C}$ Lees. ${ }^{1}$ The Edinburgh IBD Unit, Western General Hospital, Edinburgh, UK; ${ }^{2}$ NHS Greater Glasgow and Clyde, Glasgow, UK; ${ }^{3}$ NHS Tayside, Dundee, UK; ${ }^{4}$ NHS Forth Valley, Forth Valley, UK; ${ }^{5}$ NHS Fife, Kirkcaldy, UK; ${ }^{6}$ NHS Highlands, Inverness, UK

\subsection{6/gutjinl-2018-BSGAbstracts. 177}

Introduction The GEMINI trials and an increasing body of real-world data have demonstrated the effectiveness and safety of vedolizumab (VDZ) in IBD. However, there is limited available data about its effect on hospitalisations and steroid use. Our aim was to address this in a large real-world cohort of IBD patients from across Scotland.

Methods A multicenter retrospective cohort analysis of medical records was performed across 7 Scottish healthcare trusts. Primary outcomes were hospitalisation rates and overall steroid use in patients remaining on VDZ. Secondary outcomes were safety and intention to treat steroid free remission rates in patients with active disease. All data were prospectively collected as part of routine clinical care. Baseline demographics, clinical scores (HBI or Partial Mayo), faecal calprotectin (FC), endoscopy and radiology at 3, 6 and 12 months was recorded where available. Active disease was defined as endoscopic or radiographic evidence of disease or FC $>200 \mathrm{mcg} / \mathrm{g}$. Clinical remission was defined as $\mathrm{HBI}<5$ or Partial Mayo $<2$. Biochemical remission was defined as $\mathrm{FC}<200 \mathrm{mcg} / \mathrm{g}$.

Results 340 (137 UC and 203 CD) patients were included in the primary analysis with a median follow-up of 9.4 months. Hospitalisation rates per patient-year were $0.60,0.67,0.36$ and 0.16 at baseline, 3,6 and 12 months of treatment respectively. Total number of hospitalisations reduced by $52.5 \%$ from 204 (12 months prior to VDZ) to 97 (12 months after VDZ). Proportion of patients on concomitant steroids reduced from $39.7 \%$ to $16.7 \% \quad(n=332), \quad 8.1 \% \quad(n=270), \quad 9.3 \%$ $(\mathrm{n}=194)$ at 3,6 and 12 months respectively. In patients with active CD $(n=153,75.4 \%)$ steroid free clinical and steroid free biochemical remission rates were; $54.4 \%$ and $30.2 \%$ at 3 months; $47.7 \%$ and $32.1 \%$ at 6 months; $28.6 \%$ and $33.9 \%$ at 12 months. In patients with active UC $(n=112,81.8 \%)$ steroid free clinical and steroid free biochemical remission rates were; $57.4 \%$ and $40.9 \%$ at 3 months; $51.6 \%$ and $39.1 \%$ at 6 months; $37.5 \%$ and $41.2 \%$ at 12 months. Our cohort received >2066 VDZ infusions, 2 (0.6\%) patients developed infusion reactions, $9(2.6 \%)$ patients developed serious infections and $17(5.0 \%)$ serious adverse events. 